\title{
Creating and Completing Service-Learning within Medical School Curricula: From the Learner's Perspective
}

\author{
Frini Makadia,' Priya P. Mehta, ' Clayton E. Wisely, Juan E. Santiago-Torres,' Katherine Hartmann,' Mary J. Welker,' Diane Habash. ${ }^{2}$
}

\begin{abstract}
Background: This article describes a service-learning project implemented at local free clinics by students at the Ohio State University College of Medicine and identifies key factors in their success. Methods: In response to a lack of longitudinal patient-physician relationships at free clinics, the students developed an initiative linking free clinic patients with diabetes to primary care homes for longitudinal care and counseled patients on the benefits of establishing a longitudinal relationship with a primary care physician. Results: All patients counseled were linked, compared to a historical $10 \%$ linkage rate, and $78 \%$ of patients scheduling initial appointments. Five factors were identified and listed by the students as key to the project's process, success, and impact. Conclusion: Although all of these listed factors may prove difficult to replicate, this case-study serves as a model for other medical schools incorporating service-learning and exhibits that medical students can become integral portions of healthcare infrastructure.
\end{abstract}

Keywords: Community Health Services; Students, Medical; Physicians, Primary Care; Curriculum; Physician-Patient Relations. (Source: MeSH-NLM).

\section{Introduction}

About the Author: Frini Makadia is a fourth-year medical student at the ohio State University College of Medicine in Colum bus, Ohio, United States.
In the United States as well as other countries, diabetes mellitus has become a chronic condition that has been best managed with continuous, quality care. It is estimated that by 2020, more than half of the American adult population will have diabetes or pre-diabetes, ultimately escalating healthcare costs to approximately $\$ 500$ billion per year. ${ }^{1}$ The bulk of this financial burden arises from the prevalence of diabetes and the rate of complications due to poor glycemic control within notable patient populations including those of lower socioeconomic status and of minority races. Much work has been done in the US to provide an ideal model of how to best provide care for patients with chronic diabetes through primary care homes. ${ }^{2}$ This paper discusses the ability to incorporate different resources, such as medical student-driven initiatives, in aiding and alleviating the growing strain of poorly managed chronic conditions on national health resources through primary care home expansion and connection.

In 2013, the Ohio State University College of Medicine (OSUCOM) implemented the Lead, Serve, and Inspire (LSI) curriculum. ${ }^{3-6}$ The LSI curriculum emphasizes early student participation in patient care through community preceptorships and team-based projects that promote self-directed learning and development of healthcare resources and infrastructure. ${ }^{7.8}$ In LSI, first-year students complete a community project at local medical practices. ${ }^{9,10}$ Each student identifies a specific patient population and co-develops, with partnering agencies, a program addressing medical needs of this population. The following report describes the experience of a pilot version at the Physicians' Free Clinic (PFC) directed at providing patients with diabetes and no current access to primary care physicians an opportunity for longitudinal patient-physician relationships and factors leading to this program's success.

The Physicians Free Clinic, now recognized as Physicians CareConnection, is the second largest free clinic in Ohio; the PFC provides episodic primary care through urgent care, specialty care, and supportive services to patients at $200 \%$ of the Federal Poverty Level without health insurance. After meeting with stakeholders including physicians, staff, and OSUCOM faculty, students assigned to a pilot group at the PFC identified that patients with diabetes seen at the PFC required particular attention: care continuity to best address diabetic management and complications was challenging to achieve with a rotating volunteer physician schedule. An initiative was drafted by students to link patients to primary care resources. This effort was commended by stakeholders and local Patient-Centered Medical Home (PCMH) leaders as a timely endeavor due to changes in national healthcare reform and the demands accompanying it.

\section{Methods}

Students completed and analyzed a needs assessment of six PFC patients with diabetes, none of whom had a primary care physician (PCP). Inclusion criteria consisted of an existing diagnosis of Type 1 or Type 2 Diabetes Mellitus (DM), English or Spanish as primary language, and at least one prior established appointment at the PFC. Individuals less than 18 years of age were excluded.

Consequently, students created a script for a counseling session with patients which described the importance of continuing care and addressed impediments to accessing care that were highlighted by these primary discussions. Students met with

Acceptance: Aug 29, 2015

Publication: Aug 31, 2015

Process: Peer-reviewed

${ }^{1}$ Medical Student, Ohio State University College of Medicine, USA

${ }^{2}$ Chair of the Department of Family Medicine and Associate Dean of Primary Care, Ohio State University College of Medicine, USA.

Correspondence:

Frini Makadia.

Address: $370 \mathrm{~W}$ 9th Ave, Columbus, OH 43210, USA.

Email: frini.makadia@osumc.edu 
each patient privately and held a counseling session describing the benefits of PCMHs from a pre-written script.

Each patient's stage of change regarding attitudes towards seeking a PCP was assessed." During this initial encounter, subjects also completed a survey capturing information on demographics, financial and socioeconomic status, food security, social support, primary care provider use, as well as general and diabetic health.

Additionally, students expedited linkage of patients to local PCMHs by working directly with care coordinating staff at both facilities. During this time, the number of referrals, scheduled appointments, and attended appointments were noted for each patient with the aid of coordinators at each local PCMH. Students implemented this work for six weeks while tracking linkage rates and all PCP appointments (Figure 1).

\section{Results}

During the six weeks, 23 of 23 patients (100\%) were referred to a PCMH, with 18 of 23 patients (78\%) scheduling an appointment (Figure 2).

Based on the promising potential of this pilot project, the students independently continued beyond the required preceptorship and expanded their work to an additional local free clinic, an IRB approved research initiative referred to as the LINKED study, and an OSUCOM student interest group to continue counseling sessions for uninsured patients at local clinics.

Expansion allowed: (1) continued quantification of scheduling and linkage data, (2) evaluation of the impact of counseling and linkage on patient's health and perceptions of health, and (3) generation of a 3 year-sustainable student group recognized as linkage counselors.

\section{Discussion}

The pilot student group identified factors enabling success that can allow other groups to implement similar initiatives in hopes of expanding access to primary care for patients with inadequate management and attention to their chronic conditions.

\section{Teamwork and Diversity of Students}

A key component for success within this pilot initiative lay within the group itself: students had diverse backgrounds, networks of resources, and willingness to develop meaningful concepts. While this variety was originally not pre-determined, it developed into a crucial aspect of the team's productivity. Students of various programs comprised the initial pilot group including independent study program, doctorate of medicine and philosophy $(\mathrm{MD} / \mathrm{PhD})$, master of business administration (MBA), and master of science (MS) candidates. The collaboration of all members enhanced problem solving in regards to scheduling, duties, group leadership, and communication with patients, partners, and stakeholders. Dual-degree students provided skills for evidence-based and hypothesis-driven designs that enriched approaches to clinical improvement; others maintained patient-centered focus insuring that emphasis was placed on improving patient-based aspects of patient-physician relationships.

\section{Representative Needs Assessment}

Initiating a successful service-learning project must begin with a detailed needs assessment. The group utilized three different resources in doing so: guidance from faculty with pertinent experience, patient insight on diabetes-centered healthcare, and input from various PFC stakeholders on how to incorporate into the clinic's service to its patients. The group developed an adequate cultural understanding of the clinic and its patients' needs. This effective assessment ensured that the student group contributed, while remaining faithful, to the clinic's vision and future goals regarding chronic care management. These conversations yielded the ideas ultimately leading to the formation of the LINKED study and student group.

Figure 1. Linkage and Scheduling Process.

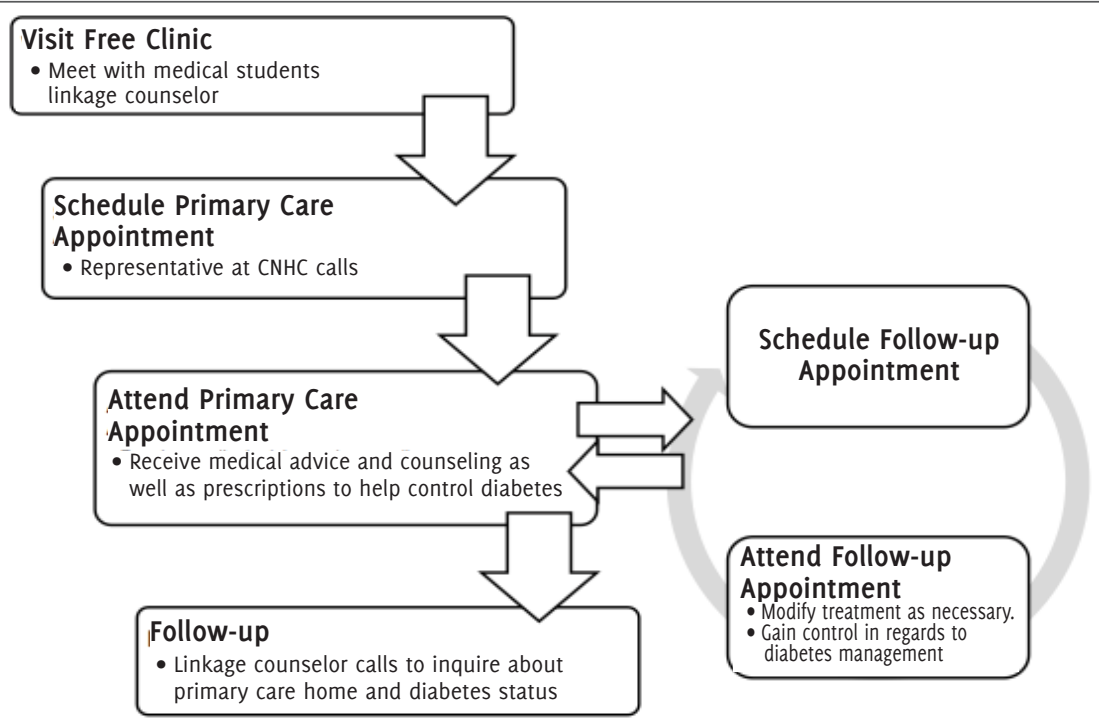

Legend: A typical clinic night begins with meeting patients and discussing the benefits of a PCP. Interested patients were put in touch with a representative that set up a primary meeting with a PCP. Patients attended an appointment, were provided information on diabetes management, and possibly scheduled follow-up appointments. 


\section{Autonomy and Sustainability}

Once trained in patient interactions and triaging procedures at the PFC, the students were given autonomy to implement and carry through the project. Counseling was integrated within the usual clinic flow and students targeted expedient linkages with PCMH partners. From years 1 to 2, students recruited and sustained the project with 6 students, later increasing to 15 , and gained recognition and funding as a student organization through the OSU Student Union and within the medical school. The success of the pilot program and its ultimate growth hinged upon this autonomy and self-sufficiency.

4. Witnessing Linkage, Health Outcomes, and Impact on Patients Sharing linkage rates with educators, physician leaders, clinic and PCMH staff yielded many positive responses. Outcomes showed a referral rate of $100 \%$ compared to $10 \%$ obtained by PFC in previous years; of those referred, $78 \%$ scheduled an initial appointment and $35 \%$ were linked to $\mathrm{PMCHs}$ with multiple, attended appointments (Figure 2). The influence of this positive feedback fueled efforts of subsequent students to expand this work along with the support of all stakeholders.

Due to this success, a research study was further drafted and approved to assess (1) linkage rates, (2) patient health before and after linkage, (3) emergency department and free clinic use before and after linkage, and (4) identification of barriers to linkage. The student's ability to quantify their initial clinical and community impact substantiated continuing community collaborations for the LSI curriculum and further highlighted the contributions medical students can provide as a mode of community resources.

\section{Right Place, Right Time}

This final factor was critical to the success of the pilot group. During the time of needs-assessment implementation, the PFC was in early stages of a paradigm shift towards linking its patients to PCMHs, but exhibited a low referral rate (10\%). The student team's success gave PFC evidence to support hiring two full-time linkage coordinators for their staff, demonstrating a fortuitously timed service by students for a community part- ner. It is not by chance that the students decided to pursue this particular project. Close coordination with clinic staff yielded directly relevant work that provided beneficial contributions beyond the boundaries of student preceptorships; this relationship provided the insight and support necessary to positively enhance clinic flow to improve patient care, support, and, potentially, long-term health.

Service-learning involves integrating academic curricula with meaningful service to enhance both aspects through formal and informal reflection. Based on the students' success and experiences in linkage, the PFC has further developed their efforts to link patients by expanding their staff to ensure adequate patient linkage - a move that relieves the financial burdens of unmanaged diabetes as well as expands the presence of the clinic in the Ohio community. The linkage process has also been an effort that health care providers and PCMHs have considered to ensure continuity of care with the same physician and improve long-term healthcare outcomes. Thus, the goals of the preliminary LINKED study align with the current reform in healthcare seen in the United States.

While this initiative has provided fruitful implementation of counseling and linkage at local free clinics, its impact is limited by sample size; the subsequent study inspired by this pilot group further addresses these concerns. Additionally, due to the nature of the patients' social infrastructure, timely and reliable communication for follow-up proved to be difficult; this was managed with strong relationships with local PMCHs which allowed for collection of linkage data.

The initiative described is one example of successful service-learning. The combination of motivated students, autonomy and flexibility, quantification of impact, and location at a facility ready for change led to successful implementation and continued expansion of the LINKED initiative. The LINKED project can be used as a model of service-learning within a medical school curriculum that aligns with current changes in national healthcare infrastructure while enhancing the local healthcare infrastructure in helpful and practical manners.

Figure 2. Initial Scheduling and Linkage Results.

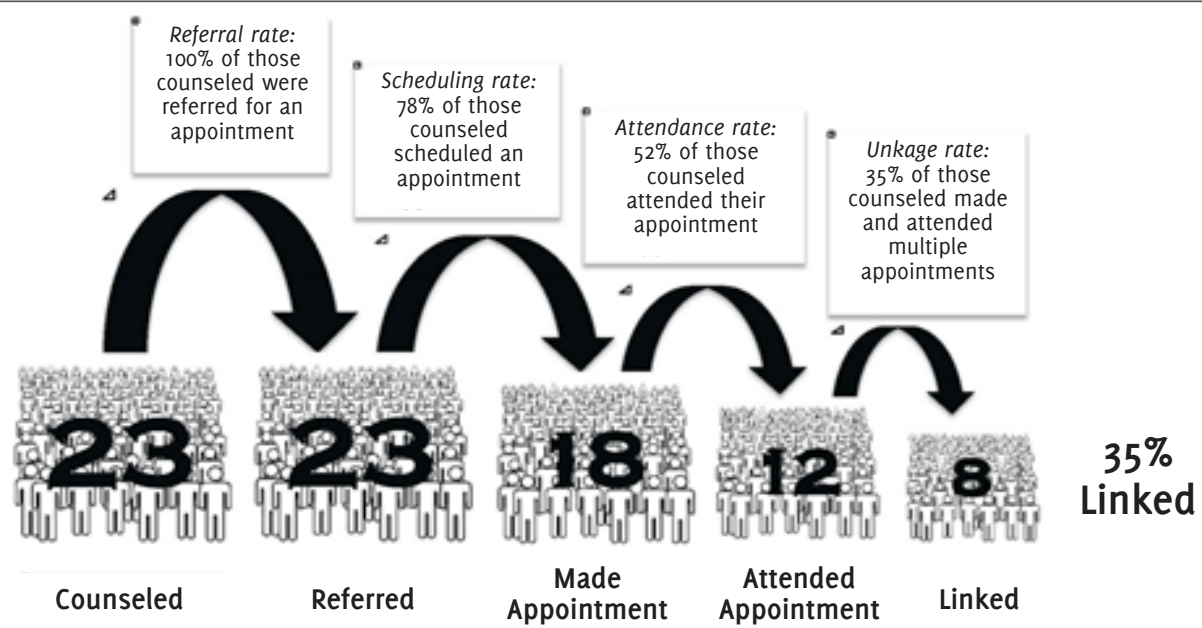

Legend: Of the original 23 patients interviewed at the PFC, 23 (100\%) were referred to PMCHs compared to the historical 10\% rate at the PFC. Eighteen patients of the original $23(78 \%)$ made an initial appointment with a primary care home and $12(52 \%)$ attended those meetings. Overall, $35 \%$ of all patients met and counseled were longitudinally linked with multiple, attended appointments with their PCP. 


\section{References}

1. United Health Group. The United States of Diabetes: challenges and opportunities in the decade ahead. 2010.

2. Horwitz DA, Schwarz ES, Scott MG, Lewis LM. Emergency department patients with diabetes have better glycemic control when they have identifiable primary care providers. Acad Emerg Med 2012 Jun;19(6):650-5.

3. Clinchot DM, Lucey CR, Gabbe SG. The Ohio State University College of Medicine. Acad Med. 2010 Sep;85(9 Suppl):S450-5.

4. Cooke M, Irby DM, O'Brien BC. Educating physicians: a call for reform of medical school and residency. San Francisco (CA): Jossey-Bass; 2010.

5. Fetterman DM, Deitz J, Gesundheit N. Empowerment evaluation: a collaborative approach to evaluating and transforming a medical school curriculum. Acad Med. 2010 May;85(5):813-20.

6. Skochelak SE. A decade of reports calling for change in medical education: what do they say? Acad Med. 2010 Sep;85(9 Suppl):S26-33.
7. Lucey CR. Medical education: part of the problem and part of the solution. JAMA Intern Med. 2013 Sep 23;173(17):1639-43.

8. Berwick DM, Finkelstein JA. Preparing medical students for the continual improvement of health and health care: Abraham Flexner and the new "public interest". Acad Med. 2010 Sep;85(9 Suppl):S56-65.

9. Institute of Medicine (US) Committee on Behavioral and Social Sciences in Medical School Curricula; Cuff PA, Vanselow NA, editors. Improving medical education: enhancing the behavioral and social science content of medical school curricula. Washington (DC): National Academies Press; 2004. p. 1-13. 10. Norris TE, Schaad DC, DeWitt D, Ogur B, Hunt DD; Consortium of Longitudinal Integrated Clerkships. Longitudinal integrated clerkships for medical students. an innovation adopted by medical schools in Australia, Canada, South Africa, and the United States. Acad Med. 2009 Jul;84(7):902-7.

11. Prochaska J0, Diclemente CC. The transtheoretical approach: crossing traditional boundaries of therapy. Homewood (IL): Dow Jones-Irwin; 1984.

\section{Acknowledgments}

The authors sincerely thank Daniel Clinchot, MD for his dedication to teaching and his guidance throughout the pilot project; the CAPS curriculum committee for the opportunity to undertake this project; Jennifer Lehman, of the Ohio State University Department of Family Medicine, for her tireless assistance with the project; and all clinics for their dedicated partnership throughout this process - including Isi Ikharebha, Executive Director of the Physicians Free Clinic (PFC), Dr. Nathan Yost, physician-volunteer at the PFC, the OSU Medical Student Steering Committee of the Columbus Free Clinic, Buhari Mohammed, MD of the Columbus Neighborhood Health Center, and Dana Vallangeon, MD of the Lower Lights Christian Health Center. The students involved in this work would also like to thank Diane Habash for her enthusiasm and dedication to both this project and our development as inquisitive, patient-focused physicians.

\section{Conflict of Interest Statement ct Funding}

The Authors have no conflicts of interest to disclose. The authors are grateful for the funding received from the Ohio State University Department of Family Medicine and the OSUMC Alumni Association

\section{Author Contributions}

Conception and design the work/idea, Collect data/obtaining results, Analysis and interpretation of data, Write the manuscript: FM, PPM, CEW, JEST, KH, MJW. Critical revision of the manuscript, Approval of the final version, Administrative or technical advice: FM, PPM, CEW, JEST, KH, MJW, DH.

Cite as:

Makadia F, Mehta PB, Wisely CE, Santiago-Torres JE, Hartmann K, Welker MJ, Habash D. Creating and Completing Service-Learning within Medical School Curricula: From the Learner's Perspective. Int J Med Students. 2015 Apr-Aug;3(2):88-91. 